\title{
Development of high spatial resolution cold/ultra- cold neutron detector using fine-grained nuclear emulsion
}

\section{N. Naganawa ${ }^{\text {a }}$}

${ }^{a}$ Institute of Materials and Systems for Sustainability, Nagoya University, Chikusa, Nagoya, 464-8602, Japan

naganawalflab.phys.nagoya-u.ac.jp

\author{
S. Awano ${ }^{b}$, M. Hino ${ }^{c}$, M. Hirose ${ }^{\text {d }}$, K. Hirota ${ }^{b}$, H. Kawaharab ${ }^{b}$ M. Kitaguchi ${ }^{b}$, \\ K. Mishima ${ }^{e}$, T. Nagae ${ }^{d}$, H. M. Shimizu ${ }^{b}$, S. Tada ${ }^{b}$, S. Tasaki ${ }^{f}$, A. Umemoto ${ }^{b}$ \\ ${ }^{b}$ Department of Physics, Nagoya University, Chikusa, Nagoya, 464-8602, Japan \\ ${ }^{c}$ Research Reactor Institute, Kyoto University, Kumatori, Osaka 590-0494, Japan \\ ${ }^{d}$ Department of Physics, Kyoto University, Kyoto 606-8502, Japan \\ ${ }^{e}$ High Energy Accelerator Research Organization, Tokai, Ibaraki 319-1106, Japan

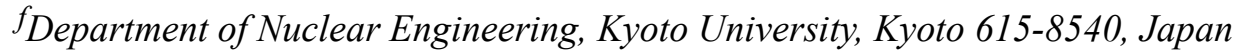

\begin{abstract}
We have been developing neutron detectors with spatial resolution of submicron to several tens of nanometers using fine-grained nuclear emulsion with nuclides with large neutron absorption cross sections such as ${ }^{6} \mathrm{Li}$ and ${ }^{10} \mathrm{~B}$. Those detectors were demonstrated their ability to detect cold and ultra-cold neutrons.
\end{abstract}

The 3rd International Symposium on "Quest for the Origin of Particles and the Universe"

5-7 January 2017

Nagoya University, Japan

\section{${ }^{1}$ Speaker}


Development of high spatial resolution cold/ultra-cold neutron detector using fine-grained nuclear emulsion,

N. Naganawa, S. Awano, M. Hino, M. Hirose, K. Hirota, H. Kawahara, M. Kitaguchi, K. Mishima, T. Nagae, H. M. Shimizu, S. Tada, S. Tasaki, A. Umemoto

\section{Introduction}

Neutron detectors with spatial resolution of submicron or less are required for experiments to search for displacement from the inverse square law in the position distribution of ultra-cold neutrons under the earth's gravitational field[1][2][3]. We have been developing such detectors by using fine-grained nuclear emulsion and nuclides with large neutron absorption cross sections emitting charged particles.

\section{Fine-grained nuclear emulsion}

Fine-grained nuclear emulsion is a kind of photographic film and works as a high spatial resolution 3 dimensional tracking detector. It consists of a substrate coated with nuclear emulsion gel containing silver bromide crystals with diameter of about $40 \mathrm{~nm}$ dispersed in gelatin[4]. When a charged particle passes through crystals in emulsion, latent images are formed in crystals. The latent images turn into aligned silver grains with diameter of about $100 \mathrm{~nm}$ by procedure of development. After the development, they can be observed by an optical microscope and recognized as a track. The fine-grained emulsion is studied and produced at Nagoya University[5]. This emulsion has no sensitivity to minimum ionizing particles, so that it has low sensitivity to gamma ray backgrounds. By using nuclides emitting large $\mathrm{dE} / \mathrm{dx}$ particles after neutron absorption, it works as a neutron detector with high $\mathrm{S} / \mathrm{N}$ ratio.

\section{Detection principle}

We used ${ }^{6} \mathrm{Li}$ and ${ }^{10} \mathrm{~B}$ as neutron absorbers. Their absorption cross sections to neutrons with velocity of 10 $\mathrm{m} / \mathrm{s}$ are large as about $2 \times 10^{5}$ barn and $8 \times 10^{5}$ barn, respectively. After absorption, they emit charged particles with large $\mathrm{dE} / \mathrm{dx}$ as equations (1) and (2).

$$
\begin{aligned}
\mathrm{n}+{ }^{6} \mathrm{Li} \rightarrow & \alpha+\mathrm{t}+4.78 \mathrm{MeV} \\
\mathrm{n}+{ }^{10} \mathrm{~B} \rightarrow & \alpha+{ }^{7} \mathrm{Li}+2.79 \mathrm{MeV}(6 \%) \\
& \alpha+{ }^{7} \mathrm{Li}+2.31 \mathrm{MeV}(94 \%)
\end{aligned}
$$

Tracks of these charged particles are detected by fine-grained nuclear emulsion. Therefore, the high spatial resolution is realized.

\section{Types of detectors}

We have been developing two types of detectors. One is called "doping type", which is nuclear emulsion gel with a chemical compound of neutron absorber doped in it. The other is "thin layer type", which consists of a thin layer of neutron absorber and a nuclear emulsion layer coated on it. In this section, we explain the principles of the two types of detectors and show the results of demonstration experiments of neutron detection.

\subsubsection{Doping type}

This type is fabricated by doping neutron absorbing nuclide into nuclear emulsion. We doped $\mathrm{LiNO}_{3}$ with natural isotope abundance of $\mathrm{Li}$ into melted gel of fine-grained nuclear emulsion up to $1.6 \times 10^{-2}$ $\mathrm{mol} / \mathrm{cm}^{3}$, and coated $1 \mathrm{~mm}$-thick glass plate. Thickness of the gel after drying was $27 \mu \mathrm{m}$ (Figure 1). When a neutron is absorbed by ${ }^{6} \mathrm{Li}$ in the emulsion, the reaction of equation (1) occurs. An $\alpha$ particle and a triton are emitted back-to-back and both of their tracks will be detected in the emulsion layer. Ranges of the $\alpha$ particle and the triton are calculated to be $7.8 \mu \mathrm{m}$ and $44 \mu \mathrm{m}$, respectively, by SRIM 2008.04. Those tracks have different $\mathrm{dE} / \mathrm{dx}$ at the starting point, therefore have different linear density of silver grains (grain density, GD). Tracks can be identified by the difference so that the starting point of tracks, i.e. neutron absorption point can be determined. 
Development of high spatial resolution cold/ultra-cold neutron detector using fine-grained nuclear emulsion,

N. Naganawa, S. Awano, M. Hino, M. Hirose, K. Hirota, H. Kawahara, M. Kitaguchi, K. Mishima, T. Nagae, H. M. Shimizu, S. Tada, S. Tasaki, A. Umemoto

\subsubsection{Demonstration experiment with neutrons}

During exposure, the detector was packed with a light-tight and airtight package made of a foil consisting of nylon $(15 \mu \mathrm{m})$, polyethylene $(13 \mu \mathrm{m})$, aluminum $(7 \mu \mathrm{m})$, polyethylene $(13 \mu \mathrm{m})$, and black polyethylene $(80 \mu \mathrm{m})$, to avoid light, since the detector being photosensitive. It was exposed to cold neutron at CN-3 beam line at Kyoto University Reactor Research Institute. After development, tracks in the detector were observed under an optical microscope with epi-illumination system. One of neutron absorption events is shown in Figure 2. A pair of tracks of an $\alpha$ particle and a triton forming a back-toback topology is seen. From GDs of $\alpha$ particles and tritons, spatial resolution of absorption points was estimated to be $0.4 \mu \mathrm{m}[6]$. Detection efficiency to cold neutron was measured at the Low Divergence Beam Branch of BL05 in MLF/J-PARC[7, 8]. It turned out to be consistent to the doped amount of Li into the detector[6].

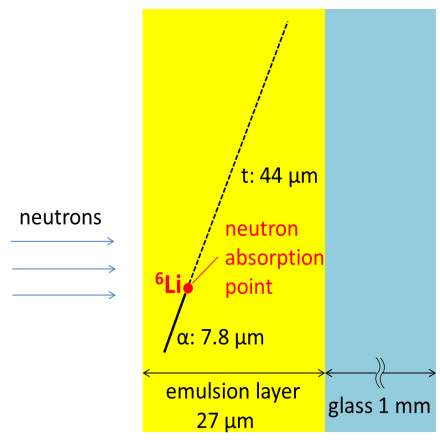

Figure 1. A cross sectional view of the doping type $\left(\mathrm{LiNO}_{3}\right)$ detector. An $\alpha$ particle and a triton emitted back-toback from neutron absorption by ${ }^{6} \mathrm{Li}$ will be detected inside the emulsion layer.

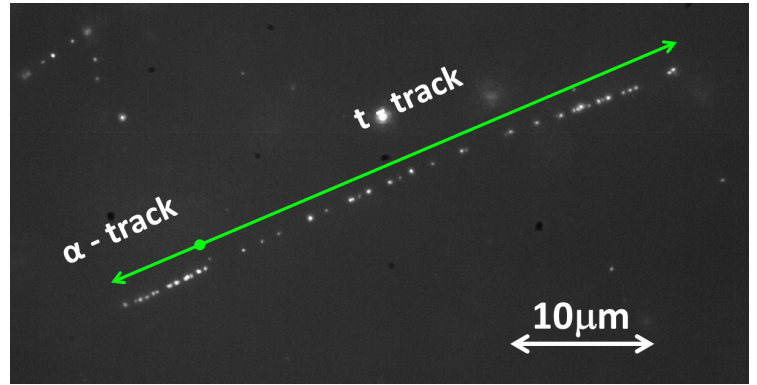

Figure 2. A microscopic view of tracks of an $\alpha$ particle and a triton emitted from neutron absorption by ${ }^{6} \mathrm{Li}$ forming a back-to-back topology. Arrows show the tracks.

\subsubsection{Thin layer type}

The other type of detector is a thin layer of neutron absorber on a substrate, coated with the fine-grained nuclear emulsion. A $50 \mathrm{~nm}$-thick $\mathrm{B}_{4} \mathrm{C}$ layer, whose ${ }^{10} \mathrm{~B}$ is enriched, was sputtered on a $400 \mu \mathrm{m}$-thick $\mathrm{Si}$ plate. Cover layers of a $60 \mathrm{~nm}$-thick NiC layer and a $30 \mathrm{~nm}$-thick $\mathrm{C}$ layer were formed on it. Finally the plate was coated with fine-grained nuclear emulsion (Figure 3 ). When a ${ }^{10} \mathrm{~B}$ absorbs a neutron, an $\alpha$ particle and a ${ }^{7} \mathrm{Li}$ are emitted back-to-back. One of them enters the emulsion layer and then will be detected. Ranges of the $\alpha$ particle and the ${ }^{7} \mathrm{Li}$ are calculated to be $5.1 \mu \mathrm{m}$ and $2.6 \mu \mathrm{m}$, respectively, by SRIM 2008.04. By extrapolating the track detected in the emulsion to the $\mathrm{B}_{4} \mathrm{C}$ layer, we can determine an absorption point with resolution depending on the thickness of the $\mathrm{B}_{4} \mathrm{C}$ layer and GDs of tracks. It is expected as several tens of nanometers for this detector.

\subsubsection{Demonstration experiment with neutrons}

The detector was exposed to cold and ultra-cold neutron, in a package made of $20 \mu \mathrm{m}$-thick aluminum foil, at BL05 in MLF. Tracks from neutron absorptions by ${ }^{10} \mathrm{~B}$ were observed under an optical microscope after development (Figure 4) for both cold and ultra-cold neutrons. 
Development of high spatial resolution cold/ultra-cold neutron detector using fine-grained nuclear emulsion,

N. Naganawa, S. Awano, M. Hino, M. Hirose, K. Hirota, H. Kawahara, M. Kitaguchi, K. Mishima, T. Nagae, H. M. Shimizu, S. Tada, S. Tasaki, A. Umemoto

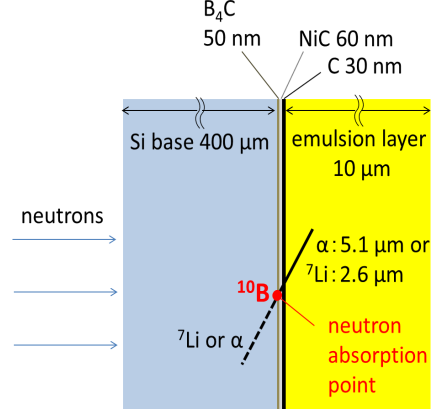

Figure 3. A cross sectional view of the thin layer $\left(B_{4} C\right)$ type detector. An $\alpha$ particle and $a^{7}$ Li are emitted back-toback from neutron absorption by ${ }^{10} B$. One of them will be detected inside the emulsion layer. The absorption point will be determined by extrapolating the track to the $B_{4}$ C layer.

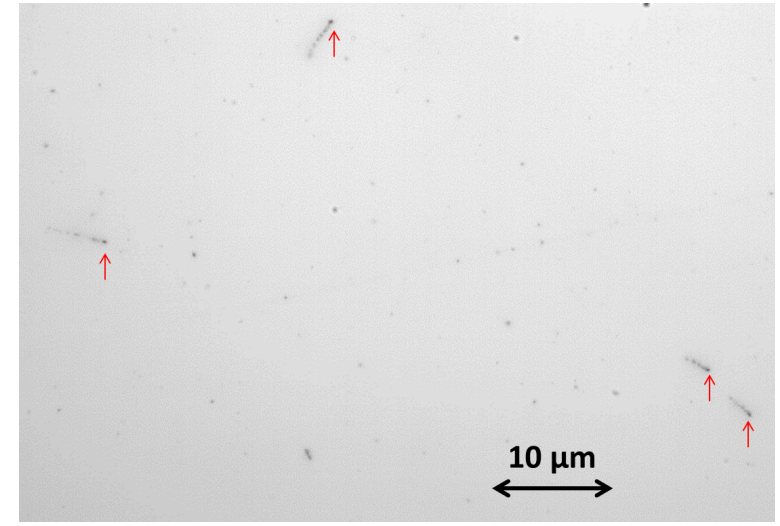

Figure 4. A microscopic view of four tracks of $\alpha$ particles or ${ }^{7} \mathrm{Li}$ s emitted from neutron absorptions by ${ }^{10}$ B. Arrows show the starting points of the tracks.

\section{Conclusion}

We have been developing cold/ultra-cold neutron detectors with spatial resolution of submicron to several tens of nanometers. There are two types of detectors: doping type and thin layer type. For doping type, $\mathrm{LiNO}_{3}$ is doped into fine-grained nuclear emulsion. Tracks from cold neutron absorptions by ${ }^{6} \mathrm{Li}$ were observed. Its spatial resolution was estimated to be $0.4 \mu \mathrm{m}$ from GDs of tracks emitted after absorptions. For thin layer type, a $50 \mathrm{~nm}$-thick $\mathrm{B}_{4} \mathrm{C}$ layer with cover layers was coated with fine-grained nuclear emulsion. Tracks from cold and ultra-cold neutron absorptions by ${ }^{10} \mathrm{~B}$ were observed. The spatial resolution of several tens of nanometers is expected to be achieved from its structure and GDs of tracks.

\section{Acknowledgements}

We acknowledge T. Naka, T. Asada, and S. Furuya for providing us fine-grained nuclear emulsion gel and advices on treatments for it. The first experimental test was conducted at CN-3 beam line at the Research Reactor Institute, Kyoto University. We acknowledge Y. Seki for sharing his beam time and helping our exposure there. We acknowledge A. Young, T. Ito, and C. Morris for test exposure at Los Alamos National Laboratory, and fruitful discussion on the structure of thin layer type. Experiments were approved by the Neutron Science Proposal Review Committee of J-PARC/MLF (Proposal No. 2014B0270, 2015A0242, and 2016A0213) and the Neutron Scattering Program Advisory Committee of IMSS, KEK (Proposal No. 2014S03). This work was supported by JSPS KAKENHI Grant Number JP26800132.

\section{References}

[1] V. V. Nesvizhevsky et al., Nature, 415, (2002).

[2] H. Abele et al., Nuclear Physics A 827 (2009) 593c-595c.

[3] G. Ichikawa et al., Phys. Rev. Lett. 112, (2014) 071101.

[4] T. Asada et al., arXiv:1705.05014 (2017)

[5] T. Naka et al., Nuclear Instruments and Methods in Physics Research A 718, (2013) 519-521.

[6] N. Naganawa et al., arXiv:1612.04544 (2016)

[7] K. Mishima et al., Nuclear Instruments and Methods in Physics Research A 600, (2009) 342-345.

[8] K. Mishima, Neutron network news (Hamon) 25 (2), (2015) 156-160. 